\title{
Lujuria de ver: literatura, género y Estado-nación en la escritura crítica de Sylvia Molloy
}

\section{Lust to see: literature, gender and nation-state in the critical writing of Sylvia Molloy}

\author{
Guadalupe MARADEI
}

Resumen: El artículo ensaya una lectura de los protocolos críticos puestos en juego en la escritura crítica de Sylvia Molloy a la hora de leer cruces entre género, modernidad y Estado-nación en corpus y debates literarios argentinos y latinoamericanos. El análisis hará foco en los textos "La cuestión del género: propuestas olvidadas y desafíos críticos" (2000); "Desvíos de lectura: sexualidad y diferencia en las letras hispanoamericanas"; "Género y modernidad" (2003) y Poses de fin de siglo. Desbordes del género en la modernidad (2012) e indagará el modo en que Molloy ha leído los cánones literarios constituidos como mecanismo de cohesión requerido por las culturas nacionales y su transgresión a partir de una lectura desviada y una relectura llamativa, particularmente atentas a las formas textuales de negación y al trabajo de las escritoras y los escritores con la pose. Molloy

Palabras clave: Literatura hispanoamericana - Crítica - Género - Estado-nación - Sylvia

Abstract: The article proposes a reading of the critical protocols that appear in the critical writing of Sylvia Molloy when she reads crossings between gender, modernity and nation-state in the literary corpus and debates of Argentine and Latin American culture. The analysis will focus on the texts "The gender issue: forgotten proposals and critical challenges" (2000); "Deviations of reading: sexuality and difference in Latin American literature"; "Gender and modernity" (2003) and End of the century poses. Overflows of gender in modernity (2012) and will investigate the way in which Molloy has read the literary canons constituted as a mechanism of cohesion required by national cultures and their transgression from a deviant reading and a striking rereading, particularly attentive to the forms textual denial and the work of writers with the pose.

Key Words: Latino American Literature - Criticism - Gender - Nation-state - Sylvia Molloy 


\section{Lectura desviada, relectura llamativa}

La eficacia escandalosa de la tetas del "Simón Bolívar" travesti que en 1994 puso en circulación el artista chileno Juan Dávila, añoró Sylvia Molloy en uno de los textos programáticos en los que imaginó las bases para una lectura crítica desde el género. Corría el año 2000 y la Revista Iberoamericana del Instituto Internacional de Literatura Iberoamericana decidió publicar un número, dirigido por Mabel Moraña, bajo el título "América Latina: agendas culturales para el Nuevo Siglo", título elocuente respecto del grado de atracción que aún detentaban los estudios culturales en la crítica literaria académica, representado también en los problemas que abordaron los textos que integraron el sumario de la publicación: "Althusser, los estudios culturales y el concepto de ideología" (Santiago Castro-Gómez), "Heterogeneidad y multiculturalismo: ¿discusión cultural o discusión legal?" (Ileana Rodríguez), "Un debate latinoamericano sobre práctica intelectual y discurso crítico" (Nelly Richard), "En torno a la crítica, la literatura, los estudios culturales y los medios masivos" (entrevista de Juan Carlos Grijalva a Françoise Perus), por mencionar algunos de sus títulos.

Dentro de ese conjunto de debates, Sylvia Molloy (la voz de una autora que ya para ese entonces había realizado aportaciones ineludibles a la crítica literaria hispanoamericana ${ }^{1}$ ) era la que sugería poner en agenda la cuestión del género. De tal modo, en la "La cuestión del género: propuestas olvidadas y desafíos críticos"2, Molloy inauguró un proceso de revisión histórica, de reflexión metacrítica y de propuesta metodológica en el que insistiría al año siguiente y hasta 2003, adquiriendo la fisonomía de un proyecto que, más tarde, actualizaría como intervención crítica y en el que conjugó un cuestionamiento radical de los modos de leer la literatura hispanoamericana con una articulación eficaz de las categorías de género, modernidad y Estado-nación.

\footnotetext{
${ }^{1}$ Además de los ensayos Las letras de Borges (1979) y Acto de presencia. La escritura autobiográfica en Hispanoamérica (1996), en ese momento ya había publicado la novela En breve cárcel y coeditado los libros Women's Writing in Latin America (1991) e Hispanism and Homosexualities (1998), algunos de cuyos ensayos fueron más tarde traducidos e incluidos en Poses de fin de siglo. Desbordes del género en la modernidad (2012).

${ }^{2}$ El ensayo fue leído originalmente en el Coloquio "Voces en conflicto, espacios de disputa", de la VI Jornada de Historia de las Mujeres y I Congreso Iberoamericano de Estudios de las Mujeres y de Género, organizado por la Facultad de Filosofía y Letras de la Universidad de Buenos Aires en agosto de 2000. En simultáneo al número mencionado de la Revista Iberoamericana, el mismo texto fue editado bajo otro título ("La flexión de género en el texto cultural latinoamericano") en la Revista de Crítica Cultural (en el año 2000) y en Cuadernos de Literatura (en 2002), lo cual indica un particular y sostenido interés en la propuesta, tanto desde los estudios culturales como desde los estudios de género, la crítica cultural y la filología.
} 


\section{Guadalupe Maradei}

"Escribir es hacer nación, no divertirse" (2000: 815), sintetiza Molloy el imperativo de Domingo Faustino Sarmiento en el prólogo a sus Viajes, imperativo cuya enunciación tenía como objeto despejar cualquier duda acerca de la "veracidad" y de la "utilidad patriótica", de las que las "ficciones de fantasía" carecían, según él, pero no su libro. Molloy juega con esa pretensión, se divierte (etimológicamente, la desvía), y lo hace a partir de una escena que -propone-constituye la cifra de los modos de leer de la crítica literaria hispanoamericana y que -avizora- en sí misma tampoco es otra cosa que un desvío.

Al comienzo del viaje a Europa que emprende en 1845, el barco en el que viajaba Sarmiento se aparta de su itinerario por accidente y recala en una isla desierta (llamada Más-afuera) en la cual encuentran una comunidad de náufragos que, el escritor exiliado se ocupa de aclarar, "estaba dividida entre sí por feudos domésticos, cuya causa no quisimos conocer, tal fue la pena que nos causó ver a estos infelices separados del resto de los hombres habitando dos cabañas a seis pasos una de la otra, ¡y sin embargo malqueriéndose y enemistados! Está visto: la discordia es una condición de nuestra existencia, aunque no haya gobierno ni mujeres..." (Sarmiento, citado en Molloy, 2000: 816).

La lectura de sesgo deconstructivo que realiza Molloy de este episodio y de las subsiguientes impresiones de Sarmiento es sugestiva y amerita la cita in extenso:

\footnotetext{
La situación parece inspirarle a Sarmiento una única reacción posible: el no preguntar acerca de la causa de esa discordia, el no querer conocer. Pero uno de esos hombres es particularmente irritante porque no respeta el silencio, habla demasiado, como una mujer. O mejor (para no hablar mal de las mujeres, dice Sarmiento), como un peluquero francés "artístico". La línea entre el silencio (del observador) y la volubilidad (del observado) se ve cruzada, cuestionada, por algo: ese algo es, precisamente, lo que no se quiere conocer (conocer la causa de la discordia conlleva el riesgo de conocer la norma de la concordia vigente) y ese algo se manifiesta, insistentemente, a través del género (aquí no literario sino sexual). Esa manifestación a través del género excede el binarismo -la discordia es de mujeres pero aquí no hay mujeres, Williams habla tanto que parece una mujer pero no es una mujer- para terminar en una representación caricatural: el peluquero francés afectado, cifra abyecta de lo otro, de un "afeminamiento" que tampoco se quiere conocer pero que se intuye suficientemente para ridiculizarlo. (Molloy, 2000: 816)
}

Molloy lee en los intersticios del relato sarmientino una escena de desvío y su posterior negación, que considera emblemática de un tipo de lectura en Hispanoamérica que consiste en no querer conocer, como Sarmiento, planteos de género, especialmente cuando vuelven reconocibles sexualidades que hacen entrar en crisis representaciones de género convencionales, cuestionando su binarismo utilitario: “...un tipo de lectura que perpetuamente desplaza el debate sobre el género y sobre la crisis de representación del género al más afuera de los proyectos de cultura nacional. (...) el desvío queda fuera de la reflexión provechosa, en el más afuera de la nación...”(2000: 817). 
La operación crítica que Molloy delinea en este texto programático y apuntala luego en otros ensayos $^{3}$ consiste en diagnosticar un punto ciego, negación o ausencia; conceptualizar el modo de leer preciso para identificar las fisuras culturales desde las cuales ejercer una lectura desviada y proponer formas de escritura crítica lo suficientemente perturbadoras para que logren provocar lo que denominó una relectura llamativa.

En el primer movimiento, Molloy señala una sistemática desestimación de la categoría de género como categoría de análisis literario ${ }^{4}$, que responde a diferentes razones. En ciertos momentos, lo asocia con un problema de jerarquías categoriales; ${ }^{5}$ en otros, con una suerte de chauvinismo teórico-crítico ${ }^{6}$; mientras que gran parte de las veces en las que despliega sus lecturas sobre objetos específicos, encuentra que la negación viene acompañada de indicios de ansiedad e incomodidad respecto de configuraciones discursivas de identidades no heteronormativas. ${ }^{7}$ Este señalamiento de Molloy entra en sintonía con las intervenciones que empezaron a propagarse en la década de 1980 a cargo de teóricas feministas de diferentes disciplinas y latitudes como Joan W. Scott. En su clásico "El género: una categoría útil para el análisis histórico", la historiadora estadounidense argumentó la necesidad de productivizar el término "género" en tanto noción relacional, sosteniendo que, si bien la atención al género no es explícita en las relaciones de poder, es una parte crucial de la organización de la igualdad o desigualdad, dado que las estructuras jerárquicas cuentan con la comprensión generalizada de la llamada relación "natural" entre varón y mujer. (Scott, 1990: 30). En la misma dirección, Molloy hace explícita su intención de "resaltar el uso productivo de la categoría de género como instrumento crítico, no sólo para proponer nuevos agrupamientos y modos de lectura sino para desautomatizar categorías que, por hábito, prescinden de él.” (Molloy, 2003: 125) y, asimismo, la

\footnotetext{
${ }^{3}$ Fundamentalmente, en "Desvíos de lectura: sexualidad y diferencia en las letras hispanoamericanas", en Estudios. Revista de Investigaciones Literarias y Culturales, Año 9, № 17, Caracas, enero-junio 2001, pp. 93-107, y en “Género y modernidad", en Herlinghaus, Hermann y Mabel Moraña (eds.), Fronteras de la modernidad en América Latina, Instituto Internacional de Literatura Iberoamericana, University of Pittsburgh, 2003.

4، Tradicionalmente el género como categoría de análisis no ha gozado de la atención ni del respeto de la crítica latinoamericana." (Molloy, 2000: 817)

5"El género, en América Latina, sigue viéndose como categoría crítica no del todo legítima, hasta abyecta, a menudo postergada cuando no subordinada a categorías consideradas más urgentes.” (Molloy, 2000: 818).

6 “...la reticencia a aceptar el género como categoría crítica tiene que ver con la consabida desconfianza de América Latina con respecto a construcciones críticas del primer mundo y mucho con una interpretación reduccionistas de la categoría 'género"'. (Molloy, 2003: 121)

7"Lo que ocurrió en esa ocasión, a mi juicio, es sintomático tanto del lugar inestable de las diferencias sexuales y de género en la literatura hispanoamericana como de la desazón que provocan en muchos lectores. Las preguntas que señalé más arriba -dónde está, y cómo leer, la y lo homosexual en el texto- afectan muchos otros textos además del mío. Porque aún esos textos provocan explicaciones, recontextualizaciones y traducciones por parte de una crítica ansiosa; provocan, por un lado, ese vaivén entre explicitación y negación que ilustraba la primera ponente de mi ejemplo o, por otro, obliteran la diferencia de género sexual mediante el desplazamiento de la discusión de género literario como era el caso de mi segundo ejemplo. (Molloy, 2001: 96)
} 


\section{Guadalupe Maradei}

necesidad de "usar el género como categoría dinámica y relacional, no como categoría estancada, para hacer lecturas no simplemente en el género sino desde el género.” (Molloy, 2003: 121).

El segundo movimiento, la conceptualización del modo de leer preciso para identificar las fisuras culturales desde las cuales ejercer una lectura desviada, se presenta demarcado por una serie de salvedades. La primera es que dicho modo de leer desde el género debe evitar la tentación del gueto, es decir, la construcción de un contrarrelato que se autoabastezca y de ese modo se naturalice al punto de parecer tan central como los mismos conjuntos que busca desestabilizar. Las construcciones a partir del género, según la autora, son siempre inestables, móviles, necesariamente ambiguas y ahí se concentra su fuerza disruptiva. La otra salvedad es que la categoría de desvío, de lectura desviada, es algo que el mismo texto, en su especificidad, suscita y no un camino que puede tomar voluntaria (o voluntaristamente) el lector. De esta forma se distancia del "uso adaptativo de Michel de Certeau" (Sarlo, 1996) que se hizo en el ámbito de las ciencias sociales latinoamericanas en la segunda mitad del siglo XX y que se centró en una celebración de los usos desviados de los textos en términos de posibilidades infinitas de apropiación y significación ("cacerías furtivas", para utilizar la metáfora de de Certeau) de los bienes culturales en circulación. La lectura desviada que propone Molloy es una lectura rara, torcida, queer, que parte de la configuración verbal del texto mismo, sin violentar su especificidad.

En el tercer movimiento, con el que condensa su programa crítico, Molloy aboga por una crítica de la literatura hispanoamericana que no sólo lea desde el género sino que además logre hacerlo de manera llamativa, que exhiba sin pudor las fisuras del texto cultural hispanoamericano y que se exhiba a sí misma, como un discurso que anhele visibilidad, desee la atención del lector y que esté a la altura de la performance del género que, asegura Molloy incorporando ahora el vocabulario teórico de Judith Butler, "siempre es un proyecto desestabilizador.” (Molloy, 2000: 819)

De esta manera, se confirma en la escritura crítica de Sylvia Molloy y particularmente en esta fase metacrítica y programática, lo mismo que Nora Domínguez percibió en su producción literaria: "La escritura de Sylvia Molloy revela que la escritura es un trabajo de producción reflexiva sobre la propia práctica (...) Su valor en la literatura argentina de mujeres consiste en escribir interpretando, en releer escribiendo." (Domínguez, 1996: 36). Y, en ese sentido, se confirma asimismo su apuesta no por una práctica crítica reproductiva sino eminentemente creativa, o por lo que Alfredo Saldaña en su ensayo "Notas para una crítica del nacionalismo cultural” llamó el "tratamiento crítico de la tradición", gracias al cual "la tradición deja de ser así un fósil y se convierte en algo vivo y dinámico, algo susceptible de ser modificado mediante la intervención activa del escritor." (Saldaña, 2008: 114) 
Advertiremos a continuación de qué manera los protocolos de lectura que encontramos expuestos de manera programática en este período de la escritura crítica de Sylvia Molloy abrevan en determinadas concepciones de la relación entre género y Estado-nación, por un lado, y de la relación entre literatura y Estado-nación, por otro, sedimentados en el período de escritura crítica inmediatamente anterior; y cuál es la relevancia, dentro de esas concepciones, de la categoría de pose que en Molloy entraña una política del cuerpo y una intervención en el espacio público.

\section{Dar a ver: el cuerpo (en) público}

“Lujuria de ver” denominó Felisberto Hernández en su relato “El acomodador” (1947) a la pasión irrefrenable del narrador personaje por observar objetos en la oscuridad, primero en su habitación, luego, a través de hendijas y/o puertas entreabiertas y, finalmente, en vitrinas de casas ajenas. La fórmula del escritor uruguayo, sostiene Molloy en uno de los ensayos que inaugura el volumen Poses de fin de siglo. Desbordes del género en la modernidad (que fue publicado en 2012 e incluye artículos escritos entre 1990 y 2001), es equiparable a la actitud de escopofilia exacerbada con la que el fin del siglo XIX procesó formas culturales estructuradas a partir de una visibilidad acrecentada. Refiere Molloy:

Todo apela a la vista y todo se espectaculariza: se exhiben nacionalidades en las exposiciones universales, se exhiben nacionalismos en los grandes desfiles militares (cuando no las guerras mismas concebidas como espectáculos), se exhiben enfermedades en los grandes hospitales, se exhibe el arte en los museos, se exhibe el sexo artístico en los 'cuadros vivos' (...) Hay exhibición y también hay exhibicionismo. (Molloy, 2012: 43)

Tal hipótesis histórica es la que habilita a la autora a proponer un modo de leer atento a ciertas escenas de voyeurismo de la cultura hispanoamericana del período que, hasta que su mirada crítica se posó sobre ellas, habían pasado inadvertidas. Se trata de escenas culturales donde colisionan, entran en tensión, se reconocen o se niegan nuevas formas de "ser en sociedad" y (lo que no es siempre lo mismo) de "ser en la nación”. El propósito, explicita Molloy, es detenerse "en ese momento de fines del siglo XIX y principios del XX en que estas formas se ven (incluso se espían) y a la vez no se nombran, ya porque carecen de nombre, ya porque quien las mira se resiste a nombrarlas." (Molloy, 2012: 12) En ese proyecto de lectura crítica va a ser decisiva la productivización que propone Molloy de la noción de pose, noción presente en todos los ensayos del libro pero fundamentada como política y deslindada como epistemología en el primer capítulo (“Clínica, nación y diferencia”). 


\section{Guadalupe Maradei}

La pose, ese trabajo escénico con el propio cuerpo en el espacio público ${ }^{8}$-que involucra el movimiento, el gesto, el vestido, la actitud y la voz- es definida hoy por la Real Academia Española como "Postura poco natural, y, por extensión, afectación en la manera de hablar y comportarse." (RAE, 2018). Esta definición, con su connotación despectiva de impostura y artificialidad, es, podría decirse, deudora del modo en que la cultura hispanoamericana del pasaje del siglo XIX al XX concibió las poses de figuras intelectuales de diversa proveniencia y notoriedad, modo que quedó registrado en relatos, crónicas, notas periodísticas, testimonios y libros recuperados y articulados como corpus por Sylvia Molloy en un intenso trabajo de archivo, lectura a contrapelo y análisis crítico en el que la cuestión del género (que poco más tarde, como vimos, sistematizaría en un proyecto programático) nunca está ausente.

Oscar Wilde es la figura paradigmática del esteticismo finisecular que verifica la fuerza desestabilizadora de la pose como gesto político. Para dar cuenta de ello, Molloy sigue de cerca escenas en las que participan como observadores José Martí, por un lado, y Rubén Darío, por el otro, con el objeto de distinguir lagunas, malentendidos y desvíos "con respecto a la decadencia europea (o lo que América Latina cree es el texto de la decadencia europea) para aprehender el significado ideológico de esas diferencias críticas.” (Molloy, 2012: 26)

La primera escena se produce en una conferencia que impartió Oscar Wilde el 7 de enero de 1882 en Nueva York. José Martí asiste como corresponsal del diario argentino La Nación. En la crónica que Martí envía al diario, si bien se valora la propuesta de Wilde en términos poéticos, Molloy detecta un primer cortocircuito cultural que no se produce a partir de otro aspecto que en la pose: la cabellera larga y el traje entallado de terciopelo inquietan al intelectual cubano a punto tal que dedica varios párrafos a describir la "singular manera" en la que Wilde viste -que no se condice con "como todos vestimos" - y a enjuiciar la elección como fuera de época. Esta contradicción entre

\footnotetext{
${ }^{8} \mathrm{Si}$ bien en los ensayos de Molloy incluidos en Poses de fin de siglo no se registra una circunscripción conceptual de las nociones de "cuerpo" y "espacio público", por los alcances que evidencian, se puede colegir que su concepción entra en sintonía con desarrollos teóricos próximos al momento de escritura de los artículos y de extendida recepción en la crítica cultural hispanoamericana. Es el caso de la noción de "tecnología política del cuerpo" que Michel Foucault acuña en Vigilar y castigar (1975) y sigue explorando en Defender la sociedad (1975-76) y en Historia de la sexualidad I. La voluntad del saber (1976). La "tecnología política del cuerpo", para Foucault, consiste en un saber del cuerpo que no es exactamente la sabiduría de su funcionamiento, y en un dominio de sus fuerzas que es más que la capacidad de vencerlas. El método genealógico que propuso Foucault en esta etapa de su producción teórica pretende interrogar los modos de surgimiento de criterios de verdad, reglamentaciones y valores, en un determinado conjunto de relaciones sociales, concebidas a su vez como relaciones de fuerzas (manifiestas o latentes); y, a la vez, analizar de qué modo ciertos ideales, regulaciones, normas y hábitos constituyen a los cuerpos individuales y colectivos. Fue Jünger Habermas, por su parte, quien en Historia y crítica de la opinión pública. La transformación estructural de la vida pública (publicado en alemán en 1962, traducido al español en 1981) historizó y caracterizó el espacio público como un territorio propio, independiente de la vida privada (aunque en constante dialéctica con ella), que no existía como tal en las sociedades medievales sino que es un producto de la Modernidad y sus instituciones.
} 
la entusiasta recepción de los preceptos artísticos y la incómoda reacción hacia la apariencia física del escritor inglés, indica, en la lectura de Molloy, un desdoblamiento semiótico: “...es otro mensaje que lo desafía, una inscripción corporal del esteticismo de fin de siglo, con un subtexto obviamente homoerótico que, como tal, lo deja perplejo.” (Molloy, 2012: 20)

La segunda escena data de diciembre de 1900 y su artífice es Rubén Darío escribiendo en París el artículo "Las purificaciones de la piedad", dedicado a Oscar Wilde, ocho días después de su muerte. El artículo comienza citando un cuento de Tolstoi que trata sobre un perro muerto encontrado en la calle, en estado descomposición. Molloy cita un fragmento de esa glosa del cuento de Tolstoi en la que se narra cómo varios transeúntes se alegran de que "esa inmundicia" haya muerto, hasta que finalmente la última voz exclama: "Sus dientes son más blancos que las finas perlas". Esa voz es adjudicada en el cuento a Jesús de Nazareth, dada la suprema piedad que manifiesta al encontrar algo que alabar en "esa fétida carroña". La analogía con la muerte del poeta homosexual no tarda en llegar: "Como un perro muerto estaba en su cuarto de soledad su miserable cadáver. En verdad sus versos y sus cuentos tienen el valor de las más finas perlas." (Darío citado en Molloy, 2012: 24)

En esta descarnada necrológica que intenta escindir la vida de la obra de un artista sin dudas admirado por Darío, Molloy conjetura que es la visibilidad de la pose de Wilde lo que está en juego:

Ese exhibicionismo, ese 'desfile caprichoso', disgusta a Darío; reprende a Wilde por no entender que 'los tiempos cambian, que Grecia antigua no es la Gran Bretaña moderna, que las psicopatías se tratan en las clínicas, que las deformidades, que las cosas monstruosas deben huir de la luz, deben tener el pudor del sol..." (Molloy, 2012: 24)

Al igual que José Martí casi dos décadas antes, Rubén Darío alude a la vida de Wilde en términos que revelan una ansiedad particular provocada por la pose y sus connotaciones. Molloy, inaugurando una utilización de categorías de la teoría psicoanalítica que será característica de todo el volumen ${ }^{9}$, entiende que Martí y Darío dan voz a una ansiedad colectiva cuya importancia ideológica es palpable. Esta ansiedad, preocupación, incomodidad, que Molloy lee en el archivo de la literatura

\footnotetext{
${ }^{9}$ Retorna, por ejemplo, cuando analiza la ansiedad que despierta la homosexualidad concretamente física en el libro de comienzos del siglo XX del subcomisario Adolfo Bastiz Buenos Aires, la ribera y los prostíbulos en 1880; cuando indaga cómo los críticos del modernismo (Octavio Paz, Carlos Fuentes) manifiestan la misma ansiedad cultural que sus objetos de estudio, al ocuparse de enfatizar la virilidad y lo fornido de aquellos escritores finiseculares; cuando registra la ansiedad que produjo en los críticos la cuidadosa construcción programática, consolidada por la elección de seudónimo que Molloy lee como su primera ficción homoerótica, por parte del escritor chileno Augusto D' Halmar; o bien, cuando señala el evidente elemento sádico presente en las prácticas de "titeo" (engaño premeditado y prolongado, desengaño y burla de víctimas seleccionadas especialmente para el caso) que llevan adelante como un divertimento habitual José Ingenieros y sus colegas de la revista Syringa. (Cfr. Molloy, 2012) Tiempo después, en los textos programáticos que ya analizamos, Molloy señalará la existencia de una "crítica ansiosa" que intenta explicar, traducir o recontextualizar el lugar inestable de las diferencias sexuales y de género en las literaturas hispanoamericanas, a través de un vaivén de explicitación/negación de una serie de sobrecompensaciones críticas (Cfr. Molloy, 2001).
} 


\section{Guadalupe Maradei}

hispanoamericana de fin de siglo XIX, va a requerir tres operaciones críticas consecuentes: una historización de la idea de pose, una epistemología de su modo de funcionamiento y una apreciación de su potencial político tanto en el momento específico de producción como en el presente de la lectura crítica.

En cuanto a la historización de la idea de pose, en el apartado "El amaneramiento voulu", Molloy precisa que, si bien no toda pose finisecular alude al homosexual (sujeto en ese momento en proceso de ser formulado legal y culturalmente), el concepto de pose connota un amaneramiento tradicionalmente marcado por lo no masculino, o por un masculino problematizado. Por lo tanto, en palabras de Molloy: “...la pose finisecular -y aquí está su aporte decisivo a la vez que su percibida amenaza- problematiza el género, su formulación y sus deslindes, subvirtiendo clasificaciones, cuestionando modelos reproductivos, proponiendo nuevos modos de identificación basados en el reconocimiento de un deseo más que en pactos culturales, invitando a (jugando a) nuevas identidades.” (Molloy, 2012: 47). En Hispanoamérica, sitúa Molloy, esa pose finisecular plantea nuevos patrones de deseo que inquietan y seducen a la vez. Por esa razón, para conjurar su posible carga transgresiva, se insiste en reducirla a una caricatura (en el caso ya mencionado de Martí y de Darío) o neutralizar su potencial ideológico viéndola como mera imitación (en el caso de José Ingenieros que incluyó la pederastia en su estudio sobre la "simulación de la locura"). De esta recepción hispanoamericana de la pose extrae Molloy una epistemología, es decir, una forma de saber, una teoría del conocimiento. ¿Qué es lo se conoce a través de la pose, según el archivo finisecular hispanoamericano? En primer lugar, la pose remite a lo no mentado, a algo cuya inscripción la constituye la pose misma: la pose, por ende, representa, es una postura significante; pero, en segundo lugar, lo no mentado, una vez inscripto y vuelto visible, se descarta ahora como pose: la pose representa (en el sentido teatral del término) pero como impostura significante. En otros términos: la pose dice que se es algo, pero decir que se es algo es posar, o sea, no serlo.

Despreciada, entonces, por vacía, caricaturizada, o integrada a un derrotero en el que figura como etapa inicial e imperfecta (aquí Molloy refiere a una lectura vigente en la época que veía la pose como etapa pasajera correspondiente a un primer modernismo de evasión, distinto de un segundo modernismo americanista, que era considerado el auténtico), la pose decadentista, tal como se puede leer en el corpus que Molloy construye, generó un amplio rechazo en la cultura hispanoamericana. Sin embargo, la autora propone otra lectura de esa pose: “...verla como gesto decisivo en la política cultural de la Hispanoamérica de fines del siglo XIX; verla, sí, como es capaz de expresar, si no 'la voz del Continente', por cierto, una de sus muchas voces, y verla precisamente como comentario de las 'inquietudes e ideales' de ese continente.” (Molloy, 2012: 42). Ese fue el 
valor histórico de la pose como modo de expresión de un aspecto de las identidades culturales en pugna. ${ }^{10}$ El valor crítico de su recuperación, tal como lo plantea Molloy en este volumen, es el germen de aquella operación-Molloy que expusimos en el apartado anterior y que consistió en conjurar el no-querer-conocer sarmientino (equivalente al no-querer-conocer cuestiones de género de la crítica literaria hispanoamericana), con una relectura llamativa. Una negación que, en este caso, Molloy expone en términos de renuncia.

En efecto, en el apartado "Pose y patología", Molloy indaga la obra de José Ingenieros como referente de la Hispanoamérica de fin de siglo que se empeñó en trabajar la pose clínicamente, como patología y como terapia a un mismo tiempo: “En el caso de Ingenieros, el desenmascaramiento de la pose (...) no lleva a la acusación sino a un desplazamiento de patologías - no es, se hace; o dicho en términos de época, no es degenerado sino simulador-, y ese desplazamiento produce una suerte de alivio cultural." (Molloy, 2012: 51) Uno de los casos estudiados por Ingenieros en La simulación de la locura (1901), y que demuestra el desplazamiento indicado por Molloy, es el de un joven literato que, encandilado por los esteticistas europeos, adopta sus todos sus vicios e incluso simula ser un "pederasta pasivo". Por fin, ante las advertencias de sus amigos, y dándose cuenta de que nadie le creía, renunció a sus "fingidas psicopatías." Este relato casuístico de Ingenieros cifra para Molloy una característica negadora y/o represiva de la crítica literaria hispanoamericana que, como vimos, luego confirmará a partir de su lectura de los Viajes de Sarmiento y que constituye el núcleo duro de su proyecto de lectura desviada de la historia de la cultura:

\begin{abstract}
El sucinto, bastante patético final del párrafo es rico en hiatos: el 'simulado' pederasta pasivo 'protesta', luego 'comprende', luego 'renuncia': nunca sabremos, a ciencia cierta, a qué. Del mismo modo creo que también 'renuncian' las culturas hispanoamericanas del fin del siglo xix a asumir esas poses que durante un brevísimo momento significaron más allá de su propia simulación. Vaciadas de pertinencia, quedaron arrumbadas, como utilería en desuso, en el closet de la representación para no hablar del closet de la crítica. Creo que era justo devolverles la llamativa visibilidad que alguna vez tuvieron. (Molloy, 2012: 53. El subrayado me pertenece.)
\end{abstract}

\title{
3. El closet de la no-nacionalidad
}

De manera complementaria a la referencia al closet de la representación y al closet de la crítica (con las que estableció, con admirable economía y sin sacarlo a relucir, un sugestivo vínculo entre literatura, crítica y la epistemología del armario que en 1990 había formulado Eve Kososvky

\footnotetext{
${ }^{10}$ No sin razón Timothy J. Reiss, en su artículo "Mapping identities: literature, nationalism, colonialism", incluye a Silvia Molloy (junto a Homi K. Bhabha, Edward W. Said, Gregory Jusdanis, Eric Cheyfitz, Terry Eagleton y Fredric Jameson) entre los/as principales críticos/as del siglo XX que reflexionaron sobre las relaciones entre literatura y nación problematizando la diferencia cultural como lo "otro" de las identidades hegemónicas. (Cfr. Reiss: 1992)
} 


\section{Guadalupe Maradei}

Sedgwick), Molloy aventura en Poses de fin de siglo la existencia de un closet de la no-nacionalidad y traza así una conexión inescindible entre género, literatura y Estado-nación. Como sostuvo Clara Kuschir, género y Estado-nación son dos realidades de muy distinta naturaleza, pero que coexisten y actúan sobre el mismo espacio físico y humano como emergentes de la convivencia social. Ambos funcionan como sistemas restrictivos, imponiendo normas, creando expectativas y estableciendo jerarquías (Kuschir, 1988). Las formulaciones culturales de esta coexistencia fueron leídas con lucidez por Sylvia Molloy en la mayor parte de su producción crítica, pero se manifiestan con particular incidencia en las secciones de Poses de fin de siglo "Pedagogía patriótica: cuerpo, género y regeneración" y "Exilios disidentes: el afuera de la patria."

En el capítulo anterior, Molloy ya había llamado la atención sobre el hecho de que en países como la Argentina, donde la población estaba transformándose vertiginosamente por efecto de una inmigración principalmente masculina, la homosexualidad pasó a ser un tema perentorio. En ese sentido, el libro de Bastiz, donde los pederastas (y, por extensión, los proxenetas, informantes, y otras figuras vinculadas al delito y a la prostitución) remiten a lo no-nacional porque "la homosexualidad existe en la Argentina, pero en realidad, viene de lejos, de Italia, de esa casa en Corso Umberto I que exporta decadentes modelos romanos a Buenos Aires” (Molloy, 2012: 31); o la inquietud que manifestaron Rubén Darío y José Martí ante la presencia de Oscar Wilde, tienen su paralelo en los discursos técnicos de los incipientes Estados-nación, que a través de distintas disciplinas intentaban definir, clasificar y analizar la desviación sexual "extranjera" como una de las enfermedades traídas por la inmigración. Este proceso, que Molloy caracteriza como una “combinación de homofobia y xenofobia", insistió en adjudicar la perversión al "afuera" con un celo que indica cuán "adentro" está en verdad ese "afuera". Pero es un proceso que no fue exclusivo de la sociedad argentina ni de Hispanoamérica sino que los propios españoles fueron partícipes en el marco de los debates por la idea de refundar el vínculo entre la metrópoli y sus ex-colonias a partir de la idea de "regeneración".

En "Semilla patriótica: regeneracionismo e inmigración", Molloy examina la "retórica del regeneracionismo español" a partir, en primer lugar, de una serie de textos testimoniales del jurista, economista, escritor y político catalán Federico Rahola (1858-1919), en los que narra su viaje a América del sur (en un momento de crisis española con la reciente y desalentadora derrota frente a Estados Unidos en Cuba), con el propósito de contribuir a la "regeneración" del comercio y la agricultura pero también con la idea de que se va a hacer o rehacer la América, en el sentido de llevar "un nuevo espíritu y nueva energía viril a las debilitadas naciones que los inmigrantes españoles reconocerán como nueva patria y donde se convertirán en hombres nuevos. 'Hacer la 
«Lujuria de ver: literatura, género y Estado-nación en la escritura crítica de Sylvia Molloy»

América' es también 'hacer(se) hombre'" (Molloy, 2012: 131). Luego de sendas analogías entre el mejoramiento de las razas vacunas y el mejoramiento de las razas humanas, en su libro Sangre nueva. Impresiones de un viaje a la América del Sud (1905), Rahola escribe textual: "Durante mi viaje he recobrado la fe en nuestra raza, convenciéndome de que tan sólo procede transformar el ambiente en que actualmente respira para hacer resurgir sus cualidades ingénitas" (Rahola citado en Molloy, 2012: 131). A continuación, no se priva de aplaudir las intervenciones de Julio Argentino Roca en tanto "representante de los antiguos capitanes y de los modernos emigrantes, que consagra definitivamente el predominio de la raza europea en la argentina" (Rahola citado en Molloy, 2012: 138). De este modo, por una parte, se intenta reposicionar a España como la nación que lleva energía física y espiritual a una región "degenerada" por la pereza y el derroche criollos y los "vicios europeos" (es decir: no españoles), y, por otra, los antiguos territorios coloniales son vistos por los intelectuales españoles como posibles fuentes de ayuda, renovación y expansión en un momento atravesado por el fracaso.

Además de la obra de Rahola, Molloy revisa los escritos del jurista, escritor y traductor ovetense Adolfo González Posada (1860-1944), en especial, su libro En América. Una campaña (1911), en donde propone la "re-siembra" de América latina como un deber nacional y redondea la actualización oportunista que el discurso regeneracionista emprendió de las nociones las abstractas de patria, raza, nación y expansión, cuando asegura "América constituye la zona natural para la expansión de nuestras ideas y de nuestros productos espirituales y naturales" (Posada citado en Molloy, 2012: 138)

Es mérito de Molloy haber dado con la reacción que obtuvo ese discurso regeneracionista entre los intelectuales vernáculos y haber yuxtapuesto algunos de sus discursos, de modo de exhibir el significativo contraste. Frente a la resiembra española, el temor a la mala semilla fue, justamente, uno de los tópicos transitados por la ficción del período, una mala semilla que en general se encontraba más asociada con la pérdida de la nacionalidad que con su consolidación. Y se presentaba también asociada con una pérdida de masculinidad. Basta con citar la afirmación de José María Ramos Mejía: "Lo que en materia de gusto y arte se le ocurre al guarango sólo un invertido puede pensarlo.” (Ramos Mejía citado en Molloy, 2012: 141).

En cada uno de los casos que releva Molloy en esta sección se confirma cómo la xenofobia y la homofobia impregnan de manera conexa los discursos de los proyectos nacionales para la modernización de América del sur que se pronunciaron desde ambos lados del Atlántico, lo cual dialoga, aportando valiosas precisiones, con las reflexiones de las teorías pos y decoloniales pero también con la preocupación sostenida que la crítica cultural ha expresado desde la década de 1980 


\section{Guadalupe Maradei}

hasta el presente por el problema de la experiencia contradictoria de la Modernidad en sus relaciones con la cultura y con el Estado-nación. En particular, esta perspectiva de Molloy anticipa la idea de "Modernidad cruel" que propuso recientemente Jean Franco (2016) como propicia para pensar la particular experiencia de ese tiempo histórico en América Latina, con su insistencia en marcar a ciertos sujetos (indígenas, mujeres, disidentes) dentro de los mismos Estados nacionales como “extraños a la modernidad” (Franco, 2016: 20).

Molloy volverá a abordar los intentos de reanimación de lo hispano en la América finisecular en su lectura de la articulación entre sexualidad e hispanismo en la producción y la trayectoria del escritor chileno Augusto D' Halmar (1882-1950). Este escritor errante y polémico, luego de fundar una comunidad masculina utópica en el sur de Chile (la Colonia Tolstoyana), emigra a España, que es para él "lugar de restauración moral y de renovación personal” (Molloy, 2012: 250) en el marco de un proceso de búsqueda que no es sólo moral sino también nacional: el "yo refaccionado" de D' Halmar se instala en una "patria renovada" (a la que a través de distintos procedimientos y traslaciones, logra adjudicarle rasgos orientales, exóticos). Sin embargo, es allí, en España, donde escribe su novela de temática homoerótica, Pasión y muerte del cura Deusto (1924), lo cual demuestra, para Molloy, por un lado, la vigencia en esos años de la reflexión hispanoamericana en torno a los orígenes y, por el otro, la inestabilidad ideológica de lo hispano, una construcción heterogénea de increíble eficacia.

En este ensayo y en el inmediatamente posterior sobre la escritora venezolana radicada en París en 1923 Teresa de la Parra (1889-1936), cobra relevancia en la lectura de Molloy la noción de exilio. A partir de la tardía publicación, en 1988, de la correspondencia cifradamente amorosa que Teresa de la Parra y Lydia Cabrera intercambiaron en la década de 1930, Molloy reconstruye cómo ambas artistas representaban "la variante latinoamericana de la lesbiana expatriada de buen pasar que elige dejar América, la del Norte o la del Sur, para llevar una vida 'independiente.”” (Molloy, 2012: 275), lo cual, sostiene, significa mucho más que la decisión circunstancial de vivir en el extranjero y debería leerse, al igual que la pose, como gesto político. En la lectura pormenorizada de esas cartas, de sus narraciones y de sus conferencias, Molloy desentraña las distintas aristas de esa “independencia”. Dos aspectos de la cosmovisión de Parra que, a simple vista pueden parecer conservadores, rescata Molloy como fuertemente disruptivos: su énfasis en la ternura en detrimento del amor y su afición por ciertos mundos anacrónicos.

En las cartas a Cabrera, Parra se manifiesta partidaria de cultivar la ternura en sus relaciones, en términos de complicidad entre personas del mismo sexo, en lugar de someterse al "combate", a la violencia, heterosexual. Esta preferencia, Molloy la lee como "estrategia de resistencia grupal 
«Lujuria de ver: literatura, género y Estado-nación en la escritura crítica de Sylvia Molloy»

opuesta al "amor", descrito como tauromaquia brutal, emblemáticamente hispánica y masculina, que es sinónimo de heterosexualidad obligatoria y por ende de un modelo social reproductivo, imperante en América Latina y sinónimo de una modernidad que Parra rechazaba.” (Molloy, 2012: 284) La ternura, entonces, se postula no como inocencia o candidez femenina sino como proyecto de liberación de formas de relación vinculadas a la heteronormatividad.

Operación análoga y atenta a las condiciones de producción del discurso, propone Molloy con los distintos alegatos de Parra en favor del mundo bucólico del siglo XIX o del claustro colonial: los interpreta como un recurso a la utopía colonial que también constituye un gesto de resistencia en la medida en que valoran una "premodernidad programática" en la que se acoge a esos "otros" que los proyectos estatales hispanoamericanos empujan hacia los márgenes y que los/as escritores/as latinoamericanos/as han problematizado en sus ficciones (los negros en Parra y en Cabrera; los campesinos y los indios en Gabriela Mistral). Esa premodernidad, añade Molloy, se inscribe en contra de una modernidad cuya taxonomía genérica y sexual no la incluye. El desplazamiento geográfico "ofrece a estas mujeres distintas lo que Venezuela, Cuba y Chile no pueden ofrecer en ese momento: esto es: un lugar para ser (sexualmente) diferente a la vez que un lugar para escribir" (Molloy, 2012: 285).

De esta forma, Sylvia Molloy contradice la tendencia a relegar a un segundo plano los aspectos internacionalistas de las prácticas literarias y produce, en cambio, una reflexión, desde la especificidad de los textos y los debates literarios hispanoamericanos, del "papel movilizador del cosmopolitismo en las literaturas nacionales" (Topuzian, 2017: 8), movilizador incluso del modo de entender las relaciones entre literatura, género y Estado-nación en un período de tiempo determinado. En esa reflexión podemos entrever en la escritura crítica de Molloy lo mismo que ella percibió en Teresa de la Parra: un prudente pero constante sesgo utópico. En la identificación de líneas de fuga de lo nacional en términos de origen y de organización de la vida, en los viajes, en los exilios y también en las comunidades reales o imaginarias (de náufragos, de escritores, de mujeres) que los/as autores/as hispanoamericanos/as han visto con deseo o turbación, Molloy sintoniza nuevamente con Judith Butler (Butler \& Spivak, 2009), esta vez, con su concepción de Estadonación y de su posibilidad de presuponer además de "modos de pertenencia" (en la medida en que sirve de matriz para los derechos y obligaciones de los/as ciudadanos/as), "modos de no-pertenencia" (desde el momento en que puede ser justamente aquello que expulsa y suspende modos de protección legal y deberes de otros/as ciudadanos/as, lo cual hace que la igualdad no sea un asunto de aumento o extensión de la homogeneidad de la nación). La apuesta de Molloy también se proyecta en la posibilidad que vislumbra Butler de concebir una democracia sensible, en la que lo nuevo irrumpa a 


\section{Guadalupe Maradei}

partir de una articulación estética dentro de la esfera política pero no en términos de construcción de un espacio imaginario, cultural, simbólico, identitario respecto del Estado-nación, sino de una contradicción performativa, una política de cambio radical que intervenga en el espacio público para demostrar, mediante esa ocupación presuntamente ilegítima, que la libertad y la igualdad pueden ir deben ir más allá de sus articulaciones existentes. Algo de eso parece haber presentido Juan Dávila cuando travistió a Bolívar en el Ministerio de Educación de Chile. Algo de eso seguro se juega en las relecturas llamativas que Sylvia Molloy nos exhibe, con programada insistencia, hace más de dos décadas.

\section{Referencias bibliográficas}

AA.VV, Diccionario de la Lengua Española, Real Academia Española, Disponible online en: http://dle.rae.es/?id=TmjCF4C [Consulta: 20/04/2018]

Butler, J., y G. Spivak (2009): ¿Quién le canta al Estado-nación? Lenguaje, política, pertenencia, Buenos Aires, Paidós.

Domínguez, N. (1994): "Identidades femeninas y literarias. Sylvia Molloy y la tradición del relato intimista", en Antelo, Raúl (org.), Identidade \& Representacao, Florianópolis, UFSC.

Franco, J. (2016): "Introducción”, en Una modernidad cruel, Ciudad de México, Fondo de Cultura Económica.

Kuschnir, C., "Género y Estado", Hiparquia, vol. I, mayo de 1988. Disponible en http://www.hiparquia.fahce.unlp.edu.ar/numeros/voli/hiparquiav1a2 [Consulta: 29/04/2018]

Maradei, G., "Crítica literaria y disidencia sexo-genérica en la cultura argentina posdictadura", Actas del IX Seminario Internacional Políticas de la Memoria, Buenos Aires, Centro Cultural de la Memoria Haroldo Conti, 2016.

Molloy, S. (2000): "La cuestión del género: propuestas olvidadas y desafíos críticos", en Revista Iberoamericana, vol. LXVI, n. ${ }^{\circ}$ 193, octubre-diciembre, 815-819. (2001): "Desvíos de lectura: sexualidad y diferencia en las letras hispanoamericanas", en Estudios. Revista de Investigaciones Literarias y Culturales, Año 9, № 17, Caracas, enerojunio, pp. 93-107.

(2003): “Género y modernidad", en Herlinghaus, Hermann y Mabel Moraña (eds.), Fronteras de la modernidad en América Latina, Instituto Internacional de Literatura Iberoamericana, University of Pittsburgh.

(2012): Poses de fin de siglo. Desbordes del género en América Latina, Buenos Aires, Eterna Cadencia.

Reiss, T. J. (1992): “Mapping Identities: Literature, Nationalism, Colonialism”, American Literary History, vol. 4, n. 4 (winter), Oxford University Press, pp. 649-677.

Saldaña, A. (2008): "Notas para una crítica del nacionalismo cultural", en L. Romero Tobar, ed., Literatura y nación: la emergencia de las literaturas nacionales, Zaragoza, Prensas Universitarias de Zaragoza, pp. 107-130.

Sarlo, B., "Retomar el debate", en Punto de vista, agosto 1996, pp.

Scott, Joan W. (1990): “El género: una categoría útil para el análisis histórico”, en James S. Amelang (ed.), Mary Nash (ed.), Historia y género: las mujeres en la Europa moderna y contemporánea, pp. 23-58 
«Lujuria de ver: literatura, género y Estado-nación en la escritura crítica de Sylvia Molloy»

Topuzián, M. (coord.), (2017): Tras la nación. Conjeturas y controversias sobre las literaturas nacionales y mundiales, Buenos Aires, EUDEBA. 